\title{
Membrana de oxigenación extracorpórea para el manejo de Shock séptico en pacientes neutropénicos con Leucemia Linfoblástica Aguda: reporte de un caso
}

Management of septic shock with extracorporeal membrane oxygenation in neutropenic patients with acute lymphoblastic leukemia: a case report

Filiación:

1 Servicio de Cirugía Cardiovascular Hospital Nacional Niños "Dr. Carlos Sáenz Herrera", San José, Costa Rica

2Servicio de Cirugía Cardiovascular Hospital Nacional Niños "Dr. Carlos Sáenz Herrera", San José, Costa Rica

3 Médico General, Universidad de Costa Rica.

4 Servicio Cirugía Cardiovascular, Hospital México, San José, Costa Rica.

Correspondencia: $\square$ José A Solano-Arce. Correo electrónico: $j_{-}$ solano_arce@hotmail.com

- - - - - - - - - -

Financiamiento:

Ninguno

Conflictos de Interés:

Ninguno

Forma de citar: Solano Arce JA, Quintana Morales R, Méndez Zamora AM, Zeledón Sánchez F. Membrana de oxigenación extracorpórea para el manejo de Shock séptico en pacientes neutropénicos con Leucemia Linfoblástica Aguda: reporte de un caso. Rev Ter. 2019;13(2):68-72.

Abreviaturas: $\quad E C M O$

Membrana de Oxigenación Extracorpórea, LLA: Leucemia Linfoblástica Aguda.

Fecha recibido: 23 de febrero 2019

Fecha aceptado: 24 mayo 2019.
José A. Solano Arce ${ }^{1}, \square$ Ronald Quintana Morales², Ana Milena Méndez Zamora ${ }^{3}$, Fernando Zeledón Sánchez ${ }^{4}$

\section{Resumen}

Se presenta el caso de un paciente masculino de 2 años portador de una leucemia linfoblástca aguda con neutropenia severa, quien mientras se encontraba en el día 22 de la fase de consolidación para el tratamiento de su leucemia, presentó un shock séptico asociado a una bronconeumonía por Pneumocystis jirovecii. El paciente ameritó terapia con membrana de oxigenación extracorpórea (por sus siglas en inglés, ECMO) como parte de su manejo y, una vez controlada la fase de shock séptico, se logró decanular al quinto día sin complicaciones para continuar su tratamiento en el servicio de hematología.

Descriptores: Shock Séptico, Membrana de oxigenación extracorpórea (ECMO), leucemia linfoblástica aguda, neutropenia febril.

\section{Abstract}

This article presents the case of a 2 year old male with acute lymphoblastic leukemia complicated with severe neutropenia that presented with septic shock due to a Pneumocystis jirovecii bronchopneumonia during the $22^{\text {nd }}$ day of the consolidation phase of his chemotherapy. His critical condition led to the patient requiring extracorporeal membrane oxygenation (ECMO). On the fifth day of ECMO, once the septic shock was resolved, the patient underwent decannulation without any complications and continued his course of treatment in the Hematology Department.

Key words: Septic shock, extracorporeal membrane oxygenation (ECMO), Acute lymphoblastic leukemia, febrile neutropenia. 


\section{Introducción}

Los procesos sépticos continúan siendo una importante causa de morbi-mortalidad en los pacientes neutropénicos en tratamiento por enfermedades malignas de estirpe hematológico. Estos alcanzan hasta un $80 \%$ de incidencia en este grupo de pacientes y alrededor de un $85 \%$ fallecen a causa de shock séptico ${ }^{1}$. Actualmente existe nueva evidencia que recomienda el uso de Membrana de Oxigenación Extracorpórea (ECMO por sus siglas en inglés), en los pacientes pediátricos con shock séptico refractario; esto debido a que en centros experimentados, si la causa del shock es secundario a una bronconeumonía, se han reportado tasas de supervivencia entre un $80-90 \%$ asociadas al manejo con $\mathrm{ECMO}^{2}$.

\section{Reporte de caso}

Se presenta el caso de un paciente masculino de 2 años de edad con diagnóstico de leucemia linfocítica aguda (LLA) diagnosticada en marzo de 2018, en tratamiento con quimioterapia en el día 22 de la fase de consolidación, con esquema de vacunación completo. Ingresa el 30 de abril del 2018, ingresa al Hospital Nacional de Niños "Dr. Carlos Sáenz Herrera" por neutropenia febril sin foco infeccioso evidente, hemograma con hemoglobina en $7.9 \mathrm{~g} /$ $\mathrm{dL}$, leucocitos 2930, con 527 neutrófilos absolutos y Proteína $C$ reactiva en 49 , examen general de orina y electrolitos normales, en vista de los hallazgos se decide iniciar cobertura antibiótica empírica con Vancomicina, Ceftazidime y Amikacina. El paciente persistió febril y asociaba datos de hipoperfusión por lo que ameritó reanimación con solución fisiológica a $20 \mathrm{cc} / \mathrm{kg}$ y tuvo una respuesta adecuada. Al tercer día de estancia, presentó deterioro del patrón respiratorio, con infiltrados bilaterales difusos en la radiografía de tórax por lo que fue necesario colocar cánula de alto flujo $(2 \mathrm{~L} / \mathrm{Kg})$, su deterioro ventilatorio fue progresivo hasta el punto de necesitar ventilación mecánica, en relación al estado clínico del paciente en ese momento, se decide cambio en la cobertura antibiótica a Meropenem y Vancomicina. Posteriormente, fue valorado por el especialista de infectología pediátrica quienes recomiendan asociar Anfotericina B y Trimetropín-sulfametoxazol a la terapia antibiótica por el alto riesgo de infección fúngica o por Pneumocystis jirovecii, se decide ampliar cobertura antibiótica de manera empírica en el contexto de un paciente con inmunosupresión secundaria al tratamiento con quimioterapia para su leucemia. Sin embargo posteriormente en un cultivo de lavado bronco-alveolar se logró aislar $P$. jirovecii y se confirma la neumonía por dicho germen.

A pesar de la antibioticoterapia y las medidas de soporte, al quinto día de estancia el paciente presentó fallo respiratorio y bradicardia, por lo que ameritó maniobras de reanimación cardiopulmonar por 10 minutos y soporte con ventilación mecánica convencional. Se trasladó a la Unidad de Cuidados Intensivos. donde se colocó en ventilación de alta frecuencia con óxido nítrico sin presentar ninguna mejoría. Para este momento, el paciente se encontraba en franco shock séptico y síndrome de distrés respiratorio agudo severo (figura 1). Debido a que el paciente cumplía con los criterios para ser colocado en soporte con membrana de oxigenación extracorpórea (ECMO) (tabla 1: pre-canulación) se decide colocar en ECMO veno-arterial de canulación central con una cánula arterial de $12 \mathrm{Fr}$ en la raíz aórtica, una cánula venosa de $18 \mathrm{Fr}$ en el atrio derecho y una membrana oxigenadora que soporta un flujo máximo de 2.5 litros por minuto (tabla 1 , post-canulación y figura 2). Se decanuló después de 117 horas de terapia ECMO cuando presentó 
mejoría clínica, radiológica (figura 3), gasometría sin hipoxemia ni hipercapnia, niveles de lactato sérico normales y reducción del soporte inotrópico (tabla 1 , pre-decanulación). Para el día 13 de mayo de 2018 es egresado de la Unidad de Cuidados Intensivos y trasladado a salón general donde completó 21 días de Trimetropim Sulfametoxazol.

El paciente fue egresado después de 26 días de estancia hospitalaria en buen estado general y con referencia para seguimiento en consulta externa del servicio de Hematología del Hospital Nacional de Niños.

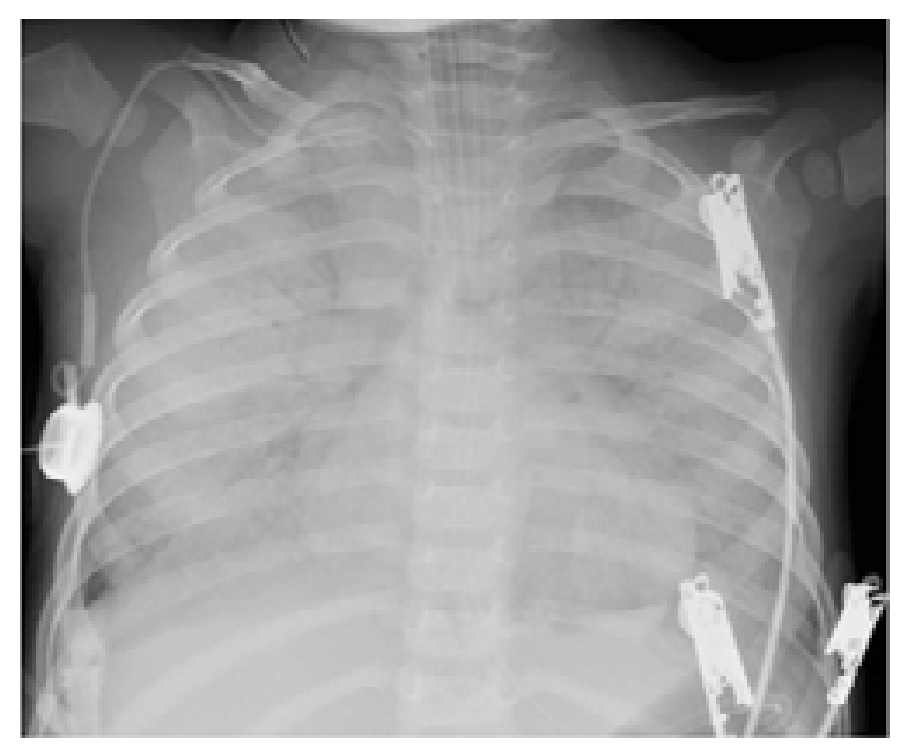

\section{Figura 1. Radiografía pre-canulación}

Radiografía pre-canulación donde se observan infiltrados difusos que comprometen los cuatro cuadrantes del parénquima pulmonar, compatible con distrés respiratorio agudo severo.

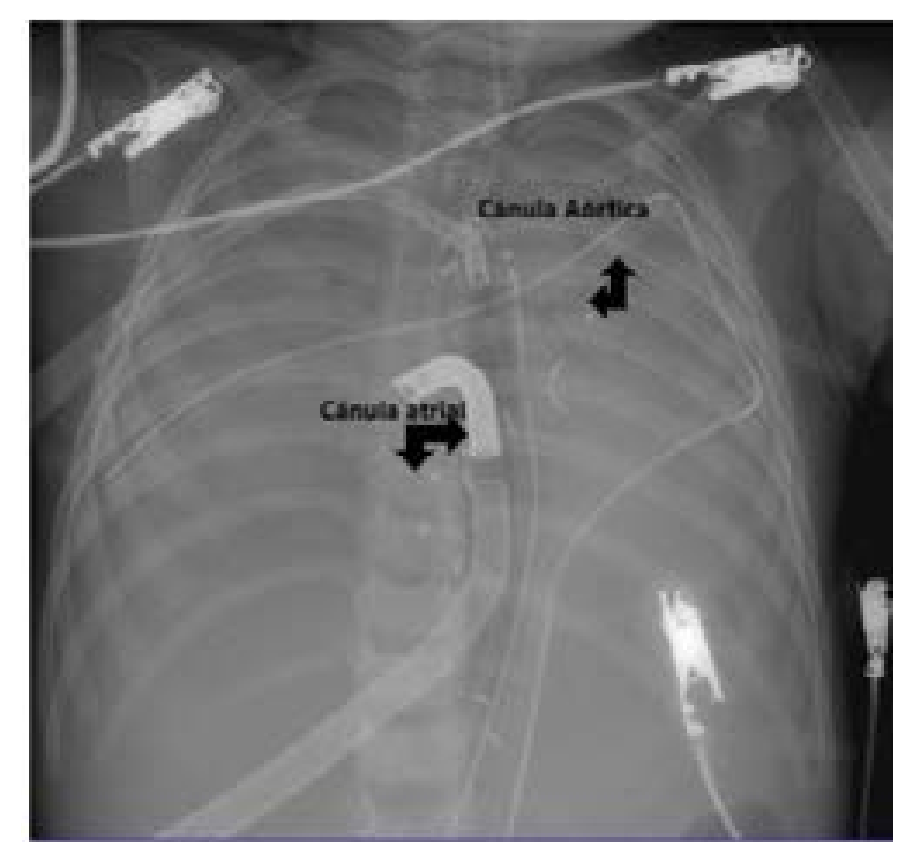

Figura 2. Radiografía post-canulación

Radiografía post-canulación donde se observa cánula venosa en atrio derecho y cánula arterial en la raíz aórtica.

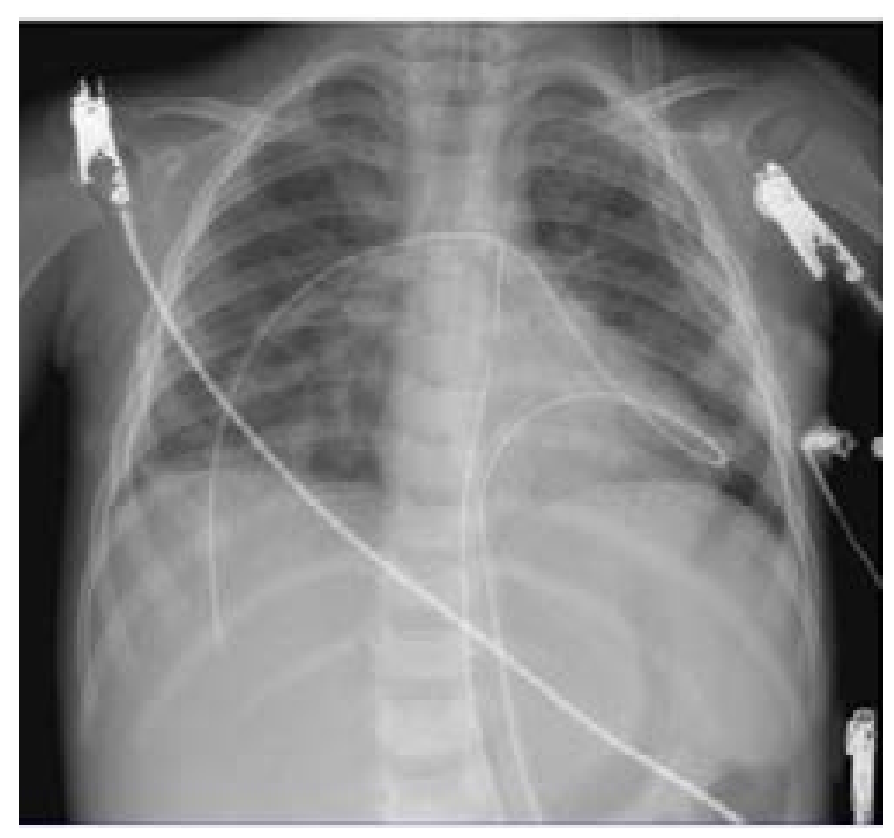

Figura 3. Radiografía post-decanulación

Radiografía post-decanulación donde observamos la ausencia de las cánulas y la resolución completa de los infiltrados pulmonares. 
Tabla 1. Evolución comparativa de Gasometría, y parámetros ventilatorios seleccionados antes, durante y después del uso de ECMO.

\begin{tabular}{|c|c|c|c|c|}
\hline $\begin{array}{c}\text { Modo de } \\
\text { Ventilación }\end{array}$ & $\begin{array}{c}\text { Pre- } \\
\text { canulación }\end{array}$ & Post-canulación & Pre-decanulación & $\begin{array}{c}\text { Post- } \\
\text { Decanulación }\end{array}$ \\
\hline $\mathrm{PMVA}$ & $22 \mathrm{cmH}_{2} \mathrm{O}$ & --- & ECMO & $\begin{array}{c}\text { Mecánica } \\
\text { convencional }\end{array}$ \\
\hline $\mathrm{FiO}_{2}$ & $100 \%$ & $100 \%$ & -- & $8 \mathrm{cmH}_{2} \mathrm{O}$ \\
\hline $\mathrm{pH}$ & 7,31 & 7,38 & $70 \%$ & $50 \%$ \\
\hline $\mathrm{PCO}_{2}$ & $48,4 \mathrm{mmHg}$ & $33 \mathrm{mmHg}$ & $41 \mathrm{mmHg}$ & 4,45 \\
\hline $\mathrm{PO}_{2}$ & $58 \mathrm{mmHg}$ & $127 \mathrm{mmHg}$ & $119 \mathrm{mmHg}$ & $160,5 \mathrm{mmHg}$ \\
\hline $\begin{array}{c}\text { Índice } \\
\text { oxigenación }\end{array}$ & 35,5 & --- & --- & 3,4 \\
\hline $\mathrm{PaO}_{2} / \mathrm{FiO}$ & $58 \mathrm{mmHg}$ & --- & --- & $320 \mathrm{mmHg}$ \\
\hline Bicarbonato & $24,2 \mathrm{mmol} / \mathrm{L}$ & $19 \mathrm{mmol} / \mathrm{L}$ & $27 \mathrm{mmol} / \mathrm{L}$ & $27 \mathrm{mmol} / \mathrm{L}$ \\
\hline Lactato & $2,6 \mathrm{mmol} / \mathrm{L}$ & $4,1 \mathrm{mmol} / \mathrm{L}$ & $<1 \mathrm{mmol} / \mathrm{L}$ & $1 \mathrm{mmol} / \mathrm{L}$ \\
\hline
\end{tabular}

$\mathrm{FiO}_{2}$ : fracción inspirada de oxígeno, PMVA: presión media de la vía aérea, $\mathrm{PCO}^{2}$ : presión parcial de dióxido de carbono, $\mathrm{PO}_{2}$ : presión parcial de oxígeno.

\section{Discusión}

Se presentó el caso de un paciente masculino de 2 años con LLA complicada con neutropenia en el que se documentó un shock séptico por Pneumocystis jirovecii. Esta infección oportunista es frecuente en portadores de LLA y tiene una incidencia de aproximadamente $40 \%$ en este tipo de pacientes. ${ }^{3} \mathrm{EI}$ proceso séptico lo llevó rápidamente a un síndrome de distrés respiratorio agudo severo manifestado por infiltrados pulmonares bilaterales difusos (Figura 1), índice de oxigenación elevado(>35) con $\mathrm{FiO}^{2}$ al $100 \%$ en ventilación de alta frecuencia (Tabla 1, pre-canulación.) y, además, asoció hipotensión refractaria que no respondió a las medidas de reanimación con fluidos ni adrenalina $(0.15 / \mathrm{mcg} /$ $\mathrm{kg} / \mathrm{min}$ ). Con base en estos parámetros el paciente cumplía criterios necesarios para ser candidato a terapia $\mathrm{ECMO}^{3,4}$. Se decidió usar la configuración veno-arterial de canulación central por los beneficios que esta aporta en pacientes con shock séptico por dos razones: primero esta configuración permite administrar soporte con flujos máximos para revertir el shock $(200 \mathrm{cc} / \mathrm{Kg} / \mathrm{min})^{5}$ (Tabla 2 ) y segundo, la administración del flujo sanguíneo arterial es directo a la aorta ascendente lo que proporciona mejor oxigenación cerebral y coronaria. ${ }^{6}$ El destete se realizó al quinto día, con soporte inotrópico mínimo, radiografía que mostraba resolución de los infiltrados (figura 3), índice de oxigenación y niveles de lactato normal (Tabla 1). 
Tabla 2. Parámetros del ECMO

\begin{tabular}{|l|l|l|}
\hline $\begin{array}{c}\text { Parámetro } \\
\text { ECMO }\end{array}$ & \multicolumn{1}{|c|}{$\begin{array}{c}\text { Post- } \\
\text { canulación }\end{array}$} & $\begin{array}{c}\text { Pre- } \\
\text { Decanulación }\end{array}$ \\
\hline Flujo & $\begin{array}{l}1,5 \mathrm{LPM}(150 \mathrm{cc} / \\
\mathrm{Kg} / \mathrm{min})\end{array}$ & $\begin{array}{l}0,68 \mathrm{LPM} \\
(68 \mathrm{cc} / \mathrm{kg})\end{array}$ \\
\hline $\mathrm{FiO}^{2}$ & $100 \%$ & $70 \%$ \\
\hline $\begin{array}{l}\text { Gas de } \\
\text { barrido }\end{array}$ & $1 \mathrm{LPM}$ & $0,6 \mathrm{LPM}$ \\
\hline
\end{tabular}

LPM: litros por minuto

\section{Conclusión}

Los resultados obtenidos en este caso clínico y la bibliografía revisada sugieren que la aplicación temprana de terapia con ECMO en pacientes con shock séptico refractario tiene un efecto positivo en la sobrevida ${ }^{7,8}$. A pesar del resultado favorable en este caso, la utilización del ECMO en shock séptico y distrés respiratorio es una indicación compleja y se necesitan de más estudios que sustenten el uso de esta terapia en tales condiciones.

\section{Referencias bibliográficas}

1. Penack O, Becker C, Buchheidt D, Christopeit M, Kiehl M, von Lilienfeld-Toal $M$, et al. Management of sepsis in neutropenic patients: 2014 updated guidelines from the Infectious Diseases Working Party of the German Society of Hematology and Medical Oncology (AGIHO). Ann Hematol. 2014;93(7):1083-1095. doi: 10.1007/ s00277-014-2086-0

2. Davis AL, Carcillo JA, Aneja RK, Deymann AJ, Lin JC, Nguyen TC, et al. American College of Critical Care Medicine Clinical Practice Parameters for Hemodynamic Support of Pediatric and Neonatal Septic Shock.
Crit Care Med. 2017;45(6):1061-1093. doi: 10.1097/CCM.0000000000002425

3. Maschmeyer G, Helweg-Larsen J, Pagano L, Robin C, Cordonnier C, Schellongowski P. ECIL guidelines for treatment of Pneumocystis jirovecii pneumonia in non-HIV-infected haematology patients. J Antimicrob Chemother. 2016;71(9):24052413. doi: $10.1093 / j a c / d k w 158$

4. Pediatric Acute Lung Injury Consensus Conference Group. Pediatric acute respiratory distress syndrome: consensus recommendations from the Pediatric Acute Lung Injury Consensus Conference. Pediatr Crit Care Med. 2015;16(5):428-439. doi: 10.1097/PCC.0000000000000350

5. Kawasaki T. Update on pediatric sepsis: a review. J Intensive Care. 2017;5:47-59. doi: 10.1186/s40560-017-0240-1

6. MacLaren G, Butt W, Best D, Donath S. Central extracorporeal membrane oxygenation for refractory pediatric septic shock. Pediatr Crit Care Med. 2011;12(2):133-136. doi: 10.1097/PCC.0b013e3181e2a4a1

7. Ruth A, McCracken CE, Fortenberry JD, Hebbar KB. Extracorporeal therapies in pediatric severe sepsis: findings from the pediatric health-care information system. Crit Care. 2015;19:397-405. doi: 10.1186/ s13054-015-1105-4

8. Brogan T, Lequier L, Lorusso, R, MacLaren G, Peek G, editores. Extracorporeal Life Support: The ELSO Red Book. 5a ed. Michigan: ELSO; 2017. 